\title{
Constitucionalización de la definición del tributo
}

\author{
Constitutionalization of tax definition
}

\author{
FRANCISCO J. RUIZ DE CASTILLA PONCE DE LEÓN* \\ CARMEN ROBLES MORENO**
}

\begin{abstract}
Resumen: El autor toma como punto de partida el proceso de constitucionalización creciente del derecho tributario de nuestro país para luego, desde la óptica de la teoría general de esta rama del derecho, realizar un análisis de la evolución del concepto de tributación. Seguidamente, nos explica la constitucionalización del derecho tributario, detallando los principales vasos comunicantes entre el derecho constitucional y el tributario actualmente. Finalmente, resalta los valores y fines constitucionales más importantes relacionados con la tributación y la aplicación del control constitucional por el Tribunal Fiscal.
\end{abstract}

Palabras clave: tributo - capacidad tributaria - tributación - derecho tributario - capacidad contributiva - política fiscal

Summary: The author works with the increasing constitutionalization process of Tax Law in the country as starting point; then, makes an analysis of tax concept evolution from a general tax law theory point of view. Afterwards, explains Tax Law Constitutionalization specifying main communicating vessels between Constitutional and Tax Law. Finally, highlights the most important values and goals related with taxing and constitutional control implementation by the Tax Court.

Key words: tax - tax capacity - taxation - tax law - capacity to pay - fiscal policy

\footnotetext{
CONTENIDO: I. SUMILLA.- $\quad$ II. CONSIDERACIONES EPISTEMOLÓGICAS.III. HISTORIA SOBRE LA DEFINICIÓN DE TRIBUTO.- IV. PRIMERA ETAPA: SIGLOS XVIII Y XIX.- IV.1. INICIOS DE LA CIENCIA ECONÓMICA.IV.2. CONOCIMIENTO ESPECIALIZADO.- IV.3. LA CAUSA DEL TRIBUTO.V.SEGUNDA ETAPA:SIGLOS XXYXXI.-V.1.REALISMO ECONÓMICO.-V.2.PRINCIPIO DE CAPACIDAD CONTRIBUTIVA.- V.3. DERECHO FINANCIERO.- V.4. DERECHO TRIBUTARIO.- V.5. DEFINICIÓN CLÁSICA DEL TRIBUTO.- V.6. PRINCIPIO DE LEGALIDAD.- V.7. CONCEPCIÓN DINÁMICA DE LA TRIBUTACIÓN.- VI. DERECHO CONSTITUCIONAL.- VI.1. POLÍTICA FISCAL Y DERECHO.- VI.2. ¿DERECHO TRIBUTARIO CONSTITUCIONAL?.- VII. CONSTITUCIONALIZACIÓN DEL DERECHO.-
}

Profesor principal de la Pontificia Universidad Católica del Perú, abogado, magíster en Derecho, correo electrónico: fruiz@ @ucp.pe

* Profesora auxiliar de la Pontificia Universidad Católica del Perú, abogada, correo electrónico: crobles@pucp.pe 
VIII. CONSTITUCIONALIZACIÓN DEL DERECHO TRIBUTARIO.- VIII.1. EL DEBER CONSTITUCIONAL.- VIII.1.1. CONCEPTO.- VIII.1.2. NATURALEZA.- VIII.1.3. ÁMBITO DEL DEBER CONSTITUCIONAL.- VIII.1.4. DEBER DE PRESTACIÓN.- VIII.2. EL DEBER DE CONTRIBUIR.-VIII.2.1.UNIVERSO.-VIII.2.3. RELACIÓN DE COOPERACIÓN ENTRE EL ESTADO Y EL CIUDADANO.- IX. CONSTITUCIONALIZACIÓN DE LA DEFINICIÓN DETRIBUTO.-IX.1.DEFINICIÓN DETRIBUTO.-IX.2.JERARQUÍA CONSTITUCIONAL.IX.3.VALORES CONSTITUCIONALES.- IX.3.1. MORAL.- IX.3.2. SOLIDARIDAD.IX.4. FINES CONSTITUCIONALES.- IX.5. SOBREINTERPRETACIÓN.- IX.6. CONTROL CONSTITUCIONAL.

\section{SUMILLA}

A partir del siglo XXI se viene intensificando en el Perú el enfoque de derecho constitucional para el desarrollo de los más diversos temas del derecho tributario. Fernando Zuzunaga del Pino ${ }^{1}$ señala que la constitucionalización del derecho tributario es un fenómeno creciente en nuestro país.

Ciertamente, la obligación tributaria es la figura central del derecho tributario. Al respecto, cada vez cobran más importancia las reflexiones constitucionales sobre la obligación tributaria. Este es precisamente el tema que abordaremos en el presente trabajo de investigación.

\section{CONSIDERACIONES EPISTEMOLÓGICAS}

Es necesario aclarar ciertos conceptos técnicos propios del trabajo de investigación científica, para facilitar la comprensión del análisis que realizaremos más adelante.

César García Novoa² sostiene que la actividad financiera del Estado consiste en el proceso de ingresos y egresos públicos. Se trata de un fenómeno (hecho que ocurre en el mundo real) estudiado por diferentes ciencias, tales como la economía (ciencia de la hacienda o política fiscal) y el derecho (derecho financiero). Específicamente el ingreso tributario es estudiado por la política fiscal y el derecho tributario.

A su vez, en el derecho debemos distinguir entre la «norma» y el «estudio de la norma». Al respecto, hay que tener presente que el ingreso tributario es regulado por normas jurídicas. Así, esta clase de reglas disciplinan la actividad del Estado en cuanto a la creación, diseño y captación del ingreso tributario. Por otra parte, Jorge Bravo $\mathrm{Cucci}^{3}$ precisa que el estudio sobre las normas jurídicas que regulan al ingreso tributario se conoce como dogmática jurídica (derecho tributario).

1 Cf. ZUZUNAGA, Fernando. ¿La cláusula antielusiva general vulnera a la Constitución peruana? Revista del Instituto Peruano de Derecho Tributario, 53 (febrero 2013), p. 23.

2 Cf. García, César. El concepto de tributo. Lima: Tax Editor, 2009, p. 29.

3 Cf. Bravo, Jorge. Fundamentos de derecho tributario. Tercera edición. Lima: Grijley, 2009, p. 153. 
Nuestra investigación ciertamente se ubica en el campo de la ciencia del derecho o dogmática jurídica. En este sentido, dentro de la familia de derecho romano-germánica, discurriremos específicamente por el campo de la teoría general del derecho tributario.

\section{HISTORIA SOBRE LA DEFINICIÓN DE TRIBUTO}

Francisco Escribano López ${ }^{4}$ afirma que en las ciencias sociales, como el derecho, una mejor comprensión de los conceptos obliga a recurrir a la historia (época en que aparece cada concepto) y la lógica (razón que explica la aparición de cada concepto).

En esta parte del trabajo comenzaremos por examinar el surgimiento y evolución de la teoría jurídica moderna sobre la obligación tributaria, hasta antes de la aparición del fenómeno de la constitucionalización del derecho. Este estudio resulta útil para entender el estado de la cuestión que actualmente viene siendo reelaborado por el actual proceso de constitucionalización del derecho tributario.

Para estos efectos adoptamos el enfoque de Eusebio González y Ernesto Lejeune $^{5}$, quienes proponen dos períodos históricos. El primer tramo comprende los siglos XVIII-XIX, donde todavía no existía la ciencia del derecho tributario. El segundo considera los siglos XX-XXI, que ya cuentan con la existencia de la ciencia del derecho tributario.

En cada una de estas etapas advertiremos la presencia de una suerte de «telón de fondo» que tiene que ver con las aproximaciones y distancias entre política fiscal y derecho (derecho financiero y derecho tributario), constituyendo las matrices que permiten apreciar el peso y contenido del enfoque jurídico — particularmente de orden constitucional- en torno a la obligación tributaria y sus aspectos conexos.

\section{PRIMERA ETAPA: SIGLOS XVIII Y XIX}

En la Edad Moderna, el estudio de las finanzas públicas estuvo a cargo de la política fiscal.

\section{IV.1. Inicios de la ciencia económica}

Para el pensamiento económicodel sigloXVIII, la atención predominante giró en torno al «sector privado». Iván Barco ${ }^{6}$ indica que los estudiosos

4 Cf. ESCRIBANO, Francisco. "Actual configuración del derecho financiero y tributario. El rol de la jurisprudencia constitucional y del Tribunal de Justicia de las comunidades europeas». En Introducción al derecho financiero y tributario. Lima: Jurista Editores, 2010, p. 17.

5 Cf. GonZÁlez, Eusebio y Ernesto LEJEUNE. Derecho tributario I. Tomo I, primera edición. Salamanca: Plaza Universitaria Ediciones, 2003, pp. 22-25.

6 Cf. BARCo, Iván. Teoría general de lo tributario. Lima: Grijley, 2009, pp. 39-40.

CONSTITUCIONALIZACIÓN DE LA

DEFINICIÓN

DEL TRIBUTO

CONSTITUTIONALIZATION OF TAX DEFINITION 
que pertenecieron a la escuela fisiocrática, tales como François Quesnay, Anne Robert Jacques Turgot, Víctor Riquetti Mirabeau, David Ricardo, Adam Smith, John Stuart Mill y Francisco Ferrara, pusieron especial atención a los asuntos que tenían que ver con las actividades comerciales de los agentes privados, que eran los encargados de crear la riqueza económica.

Por otra parte, César García Novoa ${ }^{7}$ anota que, gracias a la Revolución Francesa de 1789, el naciente Estado moderno se comienza a estructurar a partir de la organización de toda una maquinaria administrativa que se fija objetivos y medios para cumplir con el rol de dirigir a una determinada sociedad.

De este modo, temas tales como el Estado, presupuesto, impuestos y gestión administrativa empiezan a merecer ciertos estudios. Iván Barco ${ }^{8}$ destaca que estos tópicos fueron objeto de análisis durante los siglos XVII y XVIII, especialmente por los miembros de la escuela cameralista, tales como Johann Heinrich Gottlob von Justi, Heinrich Friedrich Karl von Stein, Adolf Wagner, Albert Eberhard Friedrich Schaffle y Joseph von Sonnenfels.

\section{IV.2. Conocimiento especializado}

Por otra parte, Eusebio González y Ernesto Lejeune señalan que:

[...] bien por influencia de la escuela clásica inglesa, bien por el auge del derecho administrativo a lo largo del siglo XIX, o mejor aún, por ambas cosas, el hecho cierto es que desde fines de siglo esta situación da paso a una primera especialización y las instituciones financieras comenzaron a ser estudiadas desde un punto de vista económico en los tratados de economía política (sección de hacienda pública) y desde un punto de vista jurídico, en los tratados de derecho administrativo (sección de derecho tributario) $)^{9}$.

Haciendo un balance sobre este desarrollo científico, César García Novoa hace notar el predominio de la política fiscal sobre el derecho ${ }^{10}$.

\section{IV.3. La causa del tributo}

En este marco, las preocupaciones científicas acerca del ingreso tributario apuntan a su origen y no tanto al contenido. Se considera que es propio de la actividad política y gestión pública la creación de cargas tributarias, quedando relegadas a un segundo plano las consideraciones sobre los aspectos jurídicos involucrados en este fenómeno financiero.

\footnotetext{
7 Ídem, p. 66.

8 BARCO, Iván. Ob. cit., pp. 38-39.

9 GonZÁleZ, Eusebio y Ernesto LEJEUnE. Ob. cit., p. 21

10 GaRcía, César. Ob. cit., p. 34.
} 
José Pedro Montero Traibel ${ }^{11}$ establece que en Alemania comenzó a ganar terreno la posición defendida por Mayer, quien afirmaba que el tributo se justifica en el poder político del Estado. De este modo la relación entre los agentes económicos (contribuyentes) y el Estado tiende a ser de corte vertical.

En suma, el estudio inicial sobre el tributo pertenece a la política fiscal y se preocupa especialmente por la causa de este fenómeno financiero, encontrando su justificación en el poder político.

\section{SEGUNDA ETAPA: SIGLOS XX Y XXI}

En la Edad Contemporánea aparece en escena el derecho (derecho financiero y derecho tributario) para enriquecer el estudio de las finanzas públicas.

\section{V.1. Realismo económico}

Desde inicios del siglo XX, siempre en los predios de la ciencia de la política fiscal, se advierte una reacción contra la posición de los estudiosos alemanes que acabamos de exponer.

Eusebio González y Ernesto Lejeune ${ }^{12}$ señalan que la concepción germánica sobre el tributo —entendiéndolo como la expresión de una relación de poder por parte del Estado- generó un desarrollo bastante intenso del formalismo (preocupación sobre el procedimiento para la creación de normas tributarias, sin profundizar sobre el contenido esencial de estas).

La reacción de los propios teóricos alemanes fue la adopción de la posición contraria: el realismo económico. De este modo se pasa a poner especial atención a los temas de fondo y no de forma.

Se plantea que es el economista y no el abogado experto en derecho civil quien debe estudiar las normas jurídicas que disciplinan la actividad financiera pública, pues solo de esta manera queda garantizado el análisis sustantivo del fenómeno fiscal.

\section{V.2. Principio de capacidad contributiva}

Dentro de este orden de ideas, Francisco Escribano López $z^{13}$ puntualiza que Benvenuto Griziotti es uno de los más importantes pensadores de la época que planteó la justificación del tributo a partir de una perspectiva económica, centrándola en la capacidad contributiva entendida como la aptitud económica para soportar cargas tributarias.

11 Cf. MONTERO, José. «Las doctrinas tributarias: su evolución y estado actual». Revista del Instituto 
Entonces se inicia un proceso de limitación del poder tributario, en la medida en que la facultad del Estado para crear tributos pasa a tener como parámetro de habilitación la existencia de riqueza económica por el contribuyente.

Es interesante advertir que la aparición en escena — por primera vezde la capacidad contributiva es un planteamiento que proviene de la política fiscal y no del derecho (constitucional).

En resumen, la concepción sobre el tributo - siempre perteneciente a los predios de la ciencia de la política fiscal- se enriquece con la presencia del tema de la capacidad contributiva, ubicándola como la causa del tributo.

Mientras que en la primera etapa la idea dominante es que la justificación del tributo se encuentra en la política, en la etapa prevalece el temperamento según el cual la causa del tributo más bien se ubica en la economía.

\section{V.3. Derecho financiero}

En primer lugar, vamos a referirnos a la relación entre la política fiscal y el derecho financiero. César García Novoa ${ }^{14}$ asevera que, a inicios del siglo XX, el derecho financiero comienza a madurar su proceso de independización respecto de la política fiscal.

En el ámbito académico se reconoce mayor sustantividad y relevancia al derecho financiero. En adelante, tanto la política fiscal como el derecho financiero comienzan a recorrer su propio camino por separado.

De esta manera quedan establecidas las condiciones para elaborar una definición del ingreso tributario a partir de contenidos jurídicos propios.

En segundo lugar nos vamos a concentrar en la relación existente entre el derecho administrativo y el derecho financiero. César García Novoa ${ }^{15}$ advierte que, a inicios del siglo XX, la actividad financiera del Estado moderno ya había alcanzado importantes niveles de diferenciación respecto del resto de la actividad de la administración pública, sobre todo por su carácter instrumental, en la medida en que los ingresos fiscales son los medios con los que cuenta el Estado para satisfacer las necesidades públicas. Este fue uno de los hechos que propició el nacimiento del derecho financiero y su progresiva independización respecto del derecho administrativo.

En este estadio del pensamiento nos encontramos ante un paso previo y necesario para el surgimiento del derecho tributario en cuyo seno se 
realizan los aportes — propiamente jurídicos — más significativos en torno a la definición del ingreso tributario.

\section{V.4. Derecho tributario}

Siguiendo a Gian María Micheli, Iván Barco ${ }^{16}$ señala que en el interior del derecho financiero se llega a notar la concurrencia de diversos cuerpos normativos, con diferencias bien marcadas, que dificultan la unidad de objeto de estudio que es propio de toda disciplina científica. En este sentido se toma nota que existen dos grupos de normas. En primer lugar, se encuentra un bloque de reglas que tiene que ver con los ingresos fiscales (obtención de ingresos públicos). En segundo lugar, está el bloque de reglas que regula la gestión del dinero público (gasto e inversión de los ingresos públicos).

Por otra parte, César García Novoa ${ }^{17}$ sostiene que, conforme pasa el tiempo, va quedando claro que el tributo constituye uno de los recursos más importantes para el Estado moderno.

De este modo, los estudios sobre el ingreso tributario van adquiriendo cierto nivel de trascendencia, especialidad y diferenciación respecto de los demás análisis que tienen que ver con las finanzas públicas.

Un primer antecedente sobre el particular se ubica en 1898, cuando aparece la primera monografía científico-jurídica sobre el impuesto - cuyo autor es Ranelletti - titulada Natura Giurídica dell'Imposta. En este texto se afirma que la causa del tributo es dual. En primer lugar, el tributo se justifica en la necesidad que tiene el Estado para satisfacer el interés colectivo. En segundo lugar, el tributo tiene por fundamento la capacidad contributiva.

Eusebio González y Ernesto Lejeune ${ }^{18}$ precisan que, más adelante, a mediados de la década de 1920, en Alemania comienza a tomar fuerza el planteamiento que aboga por la necesidad que las normas fiscales deben desprenderse de la tutela que sobre ellas ejercía el Código Civil.

En otras palabras, se postula la necesidad de que los ingresos tributarios tengan sus propios dispositivos legales, debiéndose dejar de lado la aplicación del Código Civil en la parte que regula a la obligación, pago, prescripción, etc.

Así, en Alemania se inician los primeros esfuerzos relativos al proceso de codificación fiscal. Heinrich Wilhelm Kruse ${ }^{19}$ afirma que el hito de partida del derecho tributario germano, asentado sobre la teoría de la 
realidad económica, se encuentra en el proyecto y posterior consagración de la Ordenanza Tributaria Alemana de 1919, que viene a constituir un Código Tributario; es decir, un cuerpo de reglas generales aplicables a todo ingreso tributario.

Todos estos esfuerzos científicos y normativos comenzaron a multiplicarse, de tal modo que - tal como sostienen Eusebio González y Ernesto Lejeune ${ }^{20}$ - a lo largo del siglo XX se ha venido forjando la autonomía del derecho tributario.

\section{V.5. Definición clásica del tributo Mauricio Alfredo Plazas Vega ${ }^{21}$ precisa que:}

[...] la réplica a la autoritaria tesis de la «relación de poder» [...] continuó en las primeras décadas del siglo XX con la elaboración de la doctrina sobre la «obligación tributaria» [...], cuyas bases se concretaron en destacar el vínculo obligacional de deudor y acreedor, que cabe predicar del contribuyente y Estado y traer al ámbito tributario las más importantes reglas y garantías que informan al derecho civil en esa materia. El nuevo discurso repara en la igualdad de las partes acreedora y deudora de los tributos [...].

Entonces, la relación entre los agentes económicos (contribuyentes) y el Estado tiende a ser de un corte más horizontal, a diferencia de la concepción vertical que estuvo en boga durante el siglo XIX.

Mauricio Alfredo Plazas Vega ${ }^{22}$ afirma que estas ideas fueron compartidas por importantes estudiosos, tales como Albert Hensel, Hans Nawasky y Ernst Blumenstein en Alemania y Suiza; Angel Donato Giannini y A. Berliri en Italia; Fernando Sainz de Bujanda en España, Mario Pugliese, Dino Jarach, Carlos María Giuliani Fonrouge, Hector B. Villegas, Sergio Francisco De la Garza, Carlos A. Mersán y Ramón Valdez Costa en América.

Eusebio González y Ernesto Lejeune ${ }^{23}$ indican que el derecho tributario continúa tomando como referencia al derecho civil — pero en el terreno de los conceptos y ya no de los dispositivos legales (Código Civil) — con la finalidad de elaborar sus propias definiciones.

Mauricio Alfredo Plazas Vega ${ }^{24}$ anota que es Giannini, uno de los pensadores más ilustres de la primera mitad del siglo XX, quien desarrolla

PlazAs Vega. «Potestad, competencia y función tributarias». En Tratado de derecho tributario. Lima: Palestra, 2003, pp. 181-182.

20 GONZÁLEZ Eusebio y LEJEUNE Ernesto. Ob. cit., pp. 17-18.

21 PLAZAS, Mauricio. «Potestad, competencia y función tributarias. Una apreciación crítica sobre la

«dinámica de los tributos». En Tratado de Derecho Tributario. Lima: Palestra, 2003, pp. 183-184.

22 Ídem, p. 184.

23 GonZÁLEZ Eusebio y Ernesto LEJEUNE. Ob. cit., p. 27.

24 PlazAs, Mauricio. Ob. cit., p. 184. 
con aguda profundidad la definición jurídica del ingreso tributario recurriendo a la figura de la «obligación»

En el entendido de que el objeto que se encuentra contenido en la obligación tributaria viene a ser una prestación de dar cierta suma de dinero a favor del Estado, entonces cabe sostener que la obligación tributaria se halla emparentada con relaciones económicas.

José Pedro Montero Traibel ${ }^{25}$ destaca que Berliri, por su parte, llega a sostener que las demás relaciones conexas al tributo (prestación pecuniaria) que se entablan entre los agentes económicos y el Estado constituyen deberes, ciertamente desprovistos de contenido patrimonial. Aquí se encuentran los deberes de inscripción en el padrón de contribuyentes de la administración tributaria, emisión de comprobantes de pago, etc.

\section{V.6. Principio de legalidad}

Por otro lado, tal como hace notar José Pedro Montero Traibel ${ }^{26}$ :

La adopción del régimen parlamentario, en el cual la supremacía se traslada del rey a las Cámaras, provoca un cambio total [...] supone que la ley será el fundamento de toda la organización estatal [...] provoca también mutaciones en la jerarquía de las fuentes del derecho, que así ve desplazar la hegemonía del reglamento a la ley.

A lo largo de la primera mitad del siglo XX se desarrolló el postulado que preconizaba que la ley es la causa de la obligación tributaria, de tal modo que el principio de legalidad deviene en pieza fundamental para el derecho tributario (no hay tributo sin ley), temperamento que también constituyó una reacción contra la tesis sobre la relación de poder que predominó en el siglo XIX.

Además, es importante destacar que en la primera mitad del siglo XX ya estaban sentados los dos principios básicos del ingreso tributario: capacidad contributiva (propuesta de la política fiscal) y el principio de legalidad (planteamiento del derecho tributario).

\section{V.7. Concepción dinámica de la tributación}

En la segunda mitad del siglo XX aparece un nuevo enfoque sobre la actividad tributaria que, en vez de centrarse en la génesis y diseño del tributo, más bien pone especial atención en la etapa aplicativa 
del tributo. Este enfoque es denominado por Álvaro Rodríguez Bereijo ${ }^{27}$ como «enfoque dinámico» de la tributación.

Mauricio Alfredo Plazas Vega ${ }^{28}$ establece que autores como M.S. Giannini, Benvenutti, Sandulli, Mielle, Allegretti, Gian Antonio Michelli, Augusto Fantozzi, Federico Mafezzoni, Renato Alessi, Antonio Fernando Basciu, José Luis Pérez de Ayala, Eusebio González García y Narciso Amorós Rica postulan una perspectiva administrativa y procesal del fenómeno tributario; donde el papel de la administración tributaria (Estado) pasa al primer plano de importancia.

José Pedro Montero Traibe ${ }^{29}$ puntualiza que esta concepción dinámica de la tributación retoma el principio de capacidad contributiva para efectos de legitimar la actividad recaudadora del Estado. En este sentido, se sostiene que el principio de capacidad contributiva lleva implícita la exigencia de su efectivización en la realidad. Se considera que los comportamientos de elusión y evasión tributaria provocan que los sujetos que poseen riqueza económica (capacidad contributiva) no tributen, correspondiendo a la administración tributaria la lucha contra estos flagelos sociales.

En perspectiva, podemos observar que en la primera mitad del siglo XX el derecho tributario se concentró en la «conducta positiva» del contribuyente y del administrado, desarrollando conceptos tales como la obligación tributaria y el deber administrativo vinculado con el tributo, respectivamente. En la segunda mitad del siglo XX, se aprecia una creciente preocupación por la «conducta negativa» del contribuyente y del administrado, poniendo especial atención a los fenómenos de elusión, evasión e informalidad tributaria.

\section{DERECHO CONSTITUCIONAL}

A título de antecedente, hemos revisado el «estado de la cuestión» sobre la dogmática del derecho tributario relativa a la definición del tributo. Seguidamente pasamos a examinar la reelaboración de esta definición, desde la perspectiva constitucional.

\section{VI.1. Política fiscal y derecho}

Desde fines del siglo XX se reacomodan las relaciones entre política fiscal y derecho (derecho financiero y derecho tributario).

Por una parte, se reafirma plenamente la «sustantividad económica» y «sustantividad jurídica» de la actividad financiera del Estado.

27 Rodríguez BereiJo, Álvaro. Introducción al estudio del derecho financiero. Madrid: Instituto de Estudios Fiscales, 1976, pp. 286-317. 
Por otro lado, Iván Barco ${ }^{30}$ asevera que un paso más se aprecia en el sentido de que se proclama la necesidad del enfoque interdisciplinario con la finalidad de lograr una comprensión más integral del fenómeno financiero público.

Un puente para aproximar la política fiscal y el derecho tiene que ver con la problemática del Estado y también la sociedad. En este sentido, se busca cierto nivel de correspondencia entre los planteamientos de la política fiscal y el derecho constitucional.

$\mathrm{Al}$ respecto, autores como César Landa Arroyo ${ }^{31}$ advierten que la economía social de mercado constituye el soporte del Estado social y democrático de derecho previsto en las constituciones.

Baldo Kresalja y César Ochoa ${ }^{32}$ sostienen que, en las primeras décadas del siglo XX, pensadores como Keynes postulan que la demanda genera oferta (el modelo económico gira sobre la base del sector privado) y que las fallas del mercado tienen que ser corregidas a través de una mayor intervención estatal.

Luego de la Segunda Guerra Mundial, con el auge del constitucionalismo, se llega a consolidar un modelo bien definido de Estado social de derecho, así denominado por el alemán Heller, en donde el poder político oficial (Estado) asume la responsabilidad del establecimiento de las condiciones mínimas que permitan el desarrollo social, sobre todo de los sectores más desprotegidos.

\section{VI.2. ¿Derecho tributario constitucional?}

Luis Durán Rojo ${ }^{33}$ precisa que, desde el inicio del proceso de autonomía del derecho tributario (primeras décadas del siglo XX), se tuvo en cuenta la importancia de las reflexiones constitucionales en torno al tributo.

César Iglesias Ferrer ${ }^{34}$ afirma que, en la medida que esta clase de estudios proviene - en esencia- del derecho constitucional, entonces esta área del conocimiento comienza a recibir la denominación de derecho constitucional tributario y su objeto de estudio tiene que ver con la facultad del Estado para crear obligaciones tributarias.

30 BARCO, Iván. Ob. cit., p. 56.

31 Cf. LANDA, César. «Los principios tributarios en la Constitución de 1993». En Temas de derecho tributario y de derecho público. Libro Homenaje a Armando Zolezzi Moller. Lima: Palestra, 2006, p. 38.

32 Cf. KResalJa, Baldo y César OchoA. Derecho constitucional económico. Lima: Fondo Editorial de la Pontificia Universidad Católica del Perú, 2009, p. 227.

33 Cf. DuRÁn, Luis. «La noción del deber constitucional de contribuir. Un estudio introductorio». En Temas de derecho tributario y de derecho público. Libro Homenaje a Armando Zolezzi Moller. Lima: Palestra, 2006, p. 52.

34 Cf. IGLESIAS, César. Derecho tributario: dogmática general de la tributación. Lima: Gaceta Jurídica, 2000, pp. 110-112.

CONSTITUCIONALIZACIÓN DE LA

DEFINICIÓN

DEL TRIBUTO

CONSTITUTIONA-

LIZATION OF TAX DEFINITION 
Luis Durán Rojo ${ }^{35}$ indica que, posteriormente, esta área del conocimiento ha incluido temas tales como derechos humanos y tributación, así como el deber de colaboración de terceros para luchar contra la evasión y elusión tributaria, de tal manera que en Europa algunos autores están rebautizando esta área del conocimiento proponiendo la denominación de derecho tributario constitucional.

Francisco Escribano López ${ }^{36}$ anota que en esta nueva perspectiva de enfoque es muy importante el aspecto metodológico, en cuya virtud, a partir de la jurisprudencia de los tribunales constitucionales, se vienen repensando los contenidos del derecho financiero y tributario.

Por nuestra parte, consideramos que los nuevos temas tributarios que vienen siendo abordados a partir de la jurisprudencia constitucional siempre tienen como eje central al Estado y no la obligación tributaria, de tal modo que preferimos que el área del conocimiento que aborda estas cuestiones continúe recibiendo la denominación de derecho constitucional tributario.

\section{CONSTITUCIONALIZACIÓN DEL DERECHO}

Paolo Comanducci ${ }^{37}$ destaca que, a partir de las últimas dos o tres décadas del siglo XX, en Europa continental y otros países del mundo se ha realizado un cambio importante del derecho, en el plano estructural, conocido como el fenómeno de la «constitucionalización» del derecho.

Josep Aguiló Regla ${ }^{38}$ hace notar los rasgos básicos del proceso de constitucionalización delorden jurídico. Solodestacamos aquellos perfiles que nos interesan para efectos del estudio sobre la constitucionalización de la definición del tributo.

En primer lugar, se tiene contemplada la garantía constitucional de la Constitución (la Constitución prevalece sobre la ley). En segundo lugar, se acepta una «sobreinterpretación» de la Constitución, esto es, que se deja de lado la interpretación literal y se favorece la interpretación extensiva, de modo que del texto constitucional se pueden extraer una gran cantidad de normas y principios implícitos. En tercer lugar, se considera que las normas constitucionales son susceptibles de ser aplicadas directamente, es decir, que los jueces pueden aplicar las normas constitucionales. En cuarto lugar, se impone un modelo de interpretación de las leyes conforme a la Constitución. 
Por su parte, Riccardo Guastini ${ }^{39}$ también establece algunos otros rasgos básicosque se encuentran presentes en el procesode constitucionalización del ordenamiento jurídico.

En primer lugar se hace notar que la legislación está condicionada por la Constitución, esto es, que el legislador no puede elegir libremente los fines a perseguir, pues solo puede preferir los fines considerados por la Constitución. En segundo lugar, se encuentra la garantía jurisdiccional de la Constitución; es decir, que existe un órgano (judicial o especial como el Tribunal Constitucional) encargado de velar por la supremacía de la Constitución respecto de la ley.

Josep Aguiló Regla ${ }^{40}$ puntualiza que el Estado social de derecho, con las características que acabamos de repasar, viene atravesando por cierto proceso de evolución que apunta hacia un «Estado constitucional».

\section{CONSTITUCIONALIZACIÓN DEL DERECHO TRIBUTARIO}

El proceso de constitucionalización del orden jurídico también viene alcanzando al derecho tributario, de tal modo que, en lo que respecta a la jurisprudencia y doctrina, se aprecia una reelaboración de los contenidos de la disciplina tributaria a partir de la perspectiva constitucional.

\section{VIII.1. El deber constitucional}

Se trata del concepto marco más directo que sirve para entender el proceso de constitucionalización de la definición de tributo.

\section{VIII.1.1. Concepto}

Álvaro Rodríguez Bereijo asevera que: «los deberes públicos, por contraposición a los derechos, son aquellas situaciones pasivas o de sujeción que se imponen a un sujeto para tutelar intereses que no son particulares suyos sino en beneficio del interés general de la comunidad $»^{41}$.

Nótese, como lo advierte Francisco Escribano López ${ }^{42}$, que el deber constitucional consiste en cierta relación que es entablada entre dos sujetos: Estado y ciudadano. Este último ya no es considerado un súbdito.

39 Cf. GuAStINI, Riccardo. "La "constitucionalización" del ordenamiento. Concepto y condiciones. En Interpretación, Estado y constitución. Lima: ARA Editores, 2010, pp. 156-158.

40 AGUILÓ REGLA, Josep. Ob. cit., p. 19.

41 RODRíGUEZ, Álvaro. «El deber de contribuir como deber constitucional: su significado jurídico» (segunda parte). Análisis Tributario, noviembre de 2008, p. 18. Lima.

42 Cf. Escribano, Francisco. «El deber de colaboración de las administraciones públicas en materia tributaria» (primera parte). Análisis Tributario, julio de 2009, p. 14.

CONSTITUCIONALIZACIÓN DE LA

DEFINICIÓN

DEL TRIBUTO

CONSTITUTIONALIZATION OF TAX DEFINITION 


\section{VIII.1.2. Naturaleza}

Luis Durán Rojo ${ }^{43}$ explica la evolución de la teoría sobre el deber constitucional. En primer lugar, en los siglos XVIII y XIX las disposiciones constitucionales que se referían a los deberes constitucionales eran consideradas proposiciones enunciativas que no tenían fuerza vinculante (meras recomendaciones).

En segundo lugar, una vez transcurridos los primeros lustros del siglo $\mathrm{XX}$, se configura el Estado social y democrático de derecho. Entonces los deberes constitucionales pasan a ser considerados mandatos dirigidos al legislador y no al ciudadano. Por tanto, es la ley la que recién crea la conducta específica que cada ciudadano queda comprometido a cumplir. Como se aprecia la figura de la «obligación» aparece vinculada con la ley y no a la Constitución. Por tanto, recién con la ley el Estado puede exigir al ciudadano una determinada conducta.

En tercer lugar, desde fines del siglo XX hasta la actualidad, nos encontramos en pleno tránsito del Estado social y democrático de derecho hacia el Estado constitucional. Ahora se considera que las disposiciones constitucionales que contienen deberes constituyen verdaderas «normas jurídicas», de tal modo que consisten en prestaciones exigibles (equiparables a las clásicas obligaciones). En este sentido, la norma constitucional que hace referencia a cierto deber es suficiente por sí misma, de tal modo que no requiere una ley para que su mandato sea vinculante.

Con relación a este último punto, Gregorio Cámara Villar, Juan Fernando López Aguilar, María Luisa Balaguer Callejón y José Antonio Montilla Martos ${ }^{44}$ señalan que existe una diferencia entre el deber y la norma. Mientras que en el deber no se encuentra una estructura normativa totalmente configurada (supuesto y consecuencia), en la norma sí apreciamos esta clase de estructura. De todos modos, tanto el deber como la norma son consideradas «normas jurídicas» en tanto consisten en proposiciones con sentido normativo, pertenecientes al sistema jurídico. Estos planteamientos se pueden advertir en la sentencia 16/1982, F.J.1 del Tribunal Constitucional de España.

\section{VIII.1.3. Ámbito del deber constitucional}

Francisco Escribano López ${ }^{45}$ sostiene que actualmente se considera que los deberes constitucionales no tienen por destinatario solamente al Estado, sino también a todos los ciudadanos, criterio que ha dejado establecido el Tribunal Constitucional de España en la STC 182/1997. 
Rubio Llorente ${ }^{46}$ citado por Rodríguez Bereijo, precisa que, en rigor, los deberes constitucionales tienen como destinatario inmediato al legislador (autor de la ley). De esta afirmación se colige que esta clase de deberes tiene como destinatario mediato al ciudadano en general (contribuyente o tercero).

\section{VIII.1.4. Deber de prestación}

Por otra parte, Álvaro Rodríguez Bereijo ${ }^{47}$ afirma que en el constitucionalismo más contemporáneo se incluyen deberes para los ciudadanos con contenido económico, tales como los «deberes de prestación», entre los que se encuentra el pago de tributos, que constituyen instrumentos para efectivizar el cumplimiento de fines constitucionales tales como la solidaridad y justicia social.

\section{VIII.2. El deber de contribuir}

Vamos a examinar especialmente aquellos temas que tienen que ver con el deber de prestación.

\section{VIII.2.1. Universo}

Luis Durán Rojo ${ }^{48}$ indica que el deber constitucional de contribuir incluye varias prestaciones. En primer lugar se encuentra la conducta de tributar (pago de tributos). En segundo lugar, aparte del clásico deber de cumplir con las declaraciones juradas determinativas de tributos, aparecen nuevos deberes que facilitan la labor de control de la administración tributaria, tales como la emisión de comprobantes de pago y la tenencia de libros de contabilidad. En tercer lugar se extienden deberes a cargo de terceros para que colaboren con el Estado en lo concerniente a la efectivización del cumplimiento de la obligación tributaria, asumiendo deberes de información o apoyo respecto de la actividad recaudadora que lleva a cabo la administración tributaria, por derivación directa del principio de solidaridad, según se desprende de la sentencia 50/1995 del Tribunal Constitucional de España.

\section{VIII.2.3. Relación de cooperación entre el Estado y el ciudadano}

Inicialmente, el Tribunal Constitucional de España en la STC 76/1990 del 26 de abril de 1990 afirmó que:

[...] no cabe, en efecto, sostener que la administración y el contribuyente se encuentran en la misma situación como si de una relación jurídicoprivada se tratara. Como antes se vio, esa pretendida igualdad resulta desmentida por el artículo $31.1 \mathrm{CE}$ que al configurar el deber tributario

CONSTITUCIONALIZACIÓN DE LA

DEFINICIÓN

DEL TRIBUTO

CONSTITUTIONALIZATION OFTAX DEFINITION 
como deber constitucional está autorizando al legislador para que, dentro de un sistema tributario justo, adopte las medidas que sean eficaces y atribuya a la administración las potestades que sean necesarias para exigir y lograr el exacto cumplimiento de sus obligaciones fiscales por parte de los contribuyentes, potestades que por ese hecho sitúan a la administración como «potentior persona» (superioridad jurídica) en una situación de superioridad sobre los contribuyentes.

En la medida en que se sostiene que el deber de contribuir implica un estado de sujeción por parte del ciudadano respecto del Estado, entonces se podría tener la impresión de que nos encontramos ante la configuración de una relación predominantemente vertical entre estos.

Sin embargo Francisco Escribano Lópe ${ }^{49}$ discrepa de esta doctrina constitucional, señalando que en el deber de contribuir más bien se destaca una relación de colaboración entre el Estado y el ciudadano.

Entonces entendemos que el punto de partida no es que el ciudadano se encuentra en cierto «punto muerto» (situación inactiva) y el Estado, tomando la iniciativa, le impone una carga tributaria (situación activa). Más bien se trata de que tanto el ciudadano como el Estado se encuentran en situaciones activas, que los llevan a tejer diversas relaciones de colaboración, entre la que se encuentra el deber de contribuir, cuyo fundamento, precisamente, descansa en el valor de solidaridad.

Posteriormente, el Tribunal Constitucional de España, en la STC 182/1997, parece haber atemperado su parecer, pues justifica la relación tributaria en el deber de contribuir (donde el Estado posee facultades delimitadas por el sistema jurídico) y no en la simple posición de superioridad del Estado.

\section{CONSTITUCIONALIZACIÓN DE LA DEFINICIÓN DE TRIBUTO}

Desde el punto de vista epistemológico, el procesamiento de la definición constitucional del tributo responde a una etapa del pensamiento científico contemporáneo donde se explora con mayor énfasis las relaciones entre el derecho constitucional y el derecho tributario. Tomando en cuenta los aportes del fenómeno de la constitucionalización del derecho tributario pasamos a formular nuestras reflexiones sobre la definición de tributo.

\section{IX.1. Definición de tributo}

Una reflexión sobre las definiciones, que se puede llevar a cabo en el terreno puramente abstracto, lleva a pensar que no existe una versión 
universal y perfecta del tributo. Más bien cada aproximación conceptual enfatiza ciertos aspectos del tributo. En este sentido, la definición constitucional de tributo no puede ser entendida como una suerte de superación cualitativa de los conceptos anteriores. Tampoco existe una definición constitucional del tributo que se pueda considerar única. Más bien en el concepto constitucional del tributo, cualquiera que sea su contenido específico, podemos encontrar la presencia de ciertos puntos que lo caracterizan

Otra reflexión, siempre en el plano abstracto, es que una definición constitucional del tributo determina su automática remisión a las respectivas reglas constitucionales. Entonces esta clase de concepto fija competencias normativas. En este sentido, si determinado ingreso público se encuentra comprendido dentro de la definición constitucional de tributo, le resultan aplicables todas las reglas constitucionales que correspondan.

Pasando al campo de los contenidos concretos del concepto de tributo conviene traer a colación la Sentencia del Tribunal Constitucional de España 76/1990 del 26 de abril de 1990 en la parte que sostiene que: «la recepción constitucional del deber de contribuir configura un mandato que vincula tanto a los poderes públicos como a los ciudadanos e incide en la naturaleza de la relación tributaria».

Con relación a este último punto (naturaleza de la relación tributaria), ocurre que el deber constitucional se refiere a una vinculación jurídica entre dos elementos de la vida social de los seres humanos: Estado y sociedad, de tal modo que un gran componente de esta relación tiene contenido político. Así sucede con el deber constitucional de contribuir, en cuya virtud a la sociedad (conformada por ciudadanos) le corresponde la responsabilidad de sustentar o financiar la actividad del Estado. Entonces la definición constitucional del tributo tiene que ver con ciertos componentes propios del derecho constitucional, tales como sociedad y Estado.

En cambio la obligación tributaria más bien denota una relación jurídica entre dos sujetos -Estado (acreedor) y personas naturales, empresas e instituciones (deudor) - cuyo contenido es económico, en la medida que tiene que ver con prestaciones básicamente dinerarias de los agentes del sector privado a favor del Estado. Por tanto la definición del tributo, anterior al proceso de constitucionalización del derecho, más bien comprende elementos del derecho tributario sustantivo, inspirados en el derecho civil, tales como acreedor, deudor y prestación.

Como se aprecia ambas definiciones son compatibles, pues no contienen elementos conflictivos que puedan generar la expulsión del sistema jurídico respecto de una de ellas. 


\section{IX.2. Jerarquía constitucional}

Es sabido que ahora la evaluación de la validez del tributo dentro del sistema jurídico se ha tornado más compleja, en la medida que este proceso ha pasado a tener dos niveles de análisis: primario y secundario.

En el nivel primario, el tributo debe cumplir con las reglas constitucionales (principio de constitucionalidad). En el nivel secundario, el tributo tiene que respetar las reglas legales (principio de legalidad).

En cada una de estas estaciones de análisis se maneja cierta definición de tributo. Al respecto es importante aclarar que no es que el concepto constitucional del tributo tenga per se un rango mayor que la definición legal del tributo. Lo que ocurre es que ambas definiciones se refieren a diversos aspectos del fenómeno tributario.

Mientras que el concepto constitucional de tributo contiene elementos que cumplen un rol de legitimación (aceptación social), en la medida que vienen a ser las condiciones necesarias, aunque no suficientes, para determinar la validez del tributo, la definición de tributo que se maneja a nivel legal posee elementos más bien técnicos (operacionales).

Por ejemplo, un impuesto a la sobreganancia minera puede contar con legitimación (principio de constitucionalidad) pero a lo mejor la redacción de la ley es confusa, pues no queda claro el ámbito de aplicación de la norma por ejemplo, generándose un conflicto con el principio de legalidad y dificultándose la aplicación del tributo.

\section{IX.3. Valores constitucionales}

Vamos a examinar la vinculación entre tributo y los valores.

\section{IX.3.1. Moral}

La definición constitucional de tributo se conecta con ciertos valores, tales como la solidaridad, justicia social, etc. Entonces, esta clase de concepto tiende un puente para entablar una conexión del tributo con la moral y los valores que esta encarna dentro del contexto del Estado social de derecho. Así, queda abierto un abanico de posibilidades, tanto extremas como intermedias.

En primer lugar podemos preguntarnos si la definición constitucional de tributo puede contemplar la afectación a la venta de drogas letales, como la pasta básica de cocaína o la venta de licores con altísimo contenido de alcohol.

Desde el punto de vista estrictamente moral parecerían no tener aceptación las conductas que son totalmente contrarias a la preservación de la vida. A su turno, un Estado social de derecho que tiene como 
eje central la dignidad de la persona humana no puede aceptar un concepto de tributo opuesto a la moral, por tanto el tributo que venimos analizando no tendría cabida.

En segundo lugar, es posible preguntarnos si la definición constitucional de tributo puede comprender la afectación a la venta de «drogas de consumo social», tales como la marihuana. Dentro del Estado social de derecho quizás se podría contemplar un concepto de tributo que legitime esta clase de gravámenes. En todo caso, se trata de una posibilidad intermedia donde seguramente existe un amplio margen para la discusión.

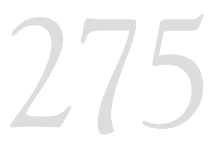

CONSTITUCIONALIZACIÓN DE LA

DEFINICIÓN

DEL TRIBUTO

CONSTITUTIONALIZATION OF TAX DEFINITION

\section{IX.3.2. Solidaridad}

Es aceptado que la definición constitucional de tributo puede contemplar deberes de prestación que no necesariamente se fundamentan en el principio de capacidad contributiva. Un caso emblemático en el Perú tiene que ver con el Impuesto a las Transacciones Financieras (ITF).

El punto de partida es que la sociedad debe financiar la actividad del Estado. Sin embargo, existe cierto sector de agentes económicos que por ejemplo se autoexcluyen del pago del Impuesto a la Renta, incurriendo en conductas de evasión tributaria cuando omiten declarar sus ingresos gravados, generándose de este modo un conflicto con el principio de solidaridad.

Ante este problema, el Estado ha reaccionado creando el ITF para que estos ciudadanos, además de realizar un pago simbólico por concepto de obligación tributaria, también cumplan con el respectivo deber de declarar a la administración tributaria sus ingresos y egresos mensuales que realizan a través del sistema bancario, por constituir las bases imponibles para la determinación de la respectiva obligación tributaria. De este modo también se facilita la fiscalización (acceso a la información bancaria sobre movimiento de fondos), y recaudación del Impuesto a la Renta por parte de la administración tributaria. En otras palabras, la creación del ITF se justifica en la medida que facilita al Estado la captación de un tributo estratégico para las finanzas públicas nacionales: el Impuesto a la Renta.

Es verdad que el movimiento bancario de los ciudadanos no necesariamente constituye una evidencia razonable de capacidad contributiva (riqueza económica), toda vez que, por ejemplo, podría tratarse del registro de importantes ingresos que ha obtenido una persona natural por concepto de una herencia recibida. En este caso no habría una riqueza económica tributable desde la perspectiva de la actual ley del Impuesto a la Renta del Perú y carecería de utilidad la aplicación del ITF. 
Sin embargo, el ITF se sustenta en la necesidad de efectivizar la solidaridad social, que viene a ser un valor fundamental que toma en cuenta la definición constitucional del tributo. Este ha sido el parecer del Tribunal Constitucional del Perú en la sentencia recaída en el expediente 0004-2004-AI/TC, FJ 39 del 21 de setiembre de 2004, en la parte que expresa lo siguiente:

Así las cosas, las afectaciones del secreto bancario que están proscritas constitucionalmente serán sólo aquellas que conlleven, en sí mismas, el propósito de quebrar la esfera íntima del individuo, mas no aquellas que, manteniendo el margen funcional del elemento de reserva que le es consustancial, sirvan a fines constitucionalmente legítimos, tales como el seguimiento de la actividad impositiva por parte de la Administración Tributaria, en aras de fiscalizar y garantizar el principio de solidaridad contributiva que le es inherente.

También es muy importante resaltar que el Tribunal Constitucional efectúa aportes sustantivos sobre el concepto constitucional de tributo, constituyendo una valiosa fuente de derecho en sentido amplio.

\section{IX.4. Fines constitucionales}

La definición constitucional del tributo se encuentra vinculada con los fines constitucionales, propios del Estado social de derecho. El desarrollo del proyecto de vida del ciudadano viene a ser uno de los fines constitucionales más importantes. Entonces, el concepto constitucional de tributo nos hace pensar que se trata de un medio para mejorar la calidad de vida de la persona humana.

Un impuesto que afecta a la venta de medicinas básicas, que son consumidas por importantes sectores sociales de bajos ingresos, no parece tener lugar dentro de la definición constitucional de tributo en la medida en que dificultaría el desarrollo del proyecto de vida de los ciudadanos.

En el Perú, el Impuesto General a las Ventas con la tasa del 18\% y el Impuesto de Promoción Municipal con la tasa del $2 \%$ afectan la venta de la gran mayoría de medicinas básicas, independientemente del nivel de ingresos del consumidor final. iEste impuesto se encuentra comprendido por la definición constitucional del tributo? Sería muy interesante que el Tribunal Constitucional del Perú se pueda pronunciar al respecto.

\section{IX.5. Sobreinterpretación}

El concepto constitucional de tributo posee un radio de cobertura bastante amplio que excede la literalidad de la norma, al punto que puede incluir ciertos casos «heterodoxos» 
Nos explicamos: la tradicional definición de obligación tributaria que se ha venido trabajando en el ámbito legal (Código Tributario) se encuentra relacionada con los pagos definitivos, es decir, con aquellas entregas de dinero que el contribuyente realiza a favor del Estado, donde —en principio - no cabe la devolución. Este es el caso del pago de regularización del Impuesto a la Renta anual que realizan las personas naturales y jurídicas.

Por otra parte, los pagos anticipados mensuales — que al final del año son compensables contra el Impuesto a la Renta anual_ — constituyen pagos provisionales, de tal modo que si el total de pagos mensuales realizados a lo largo de un ejercicio gravable suma 100 y el respectivo Impuesto a la Renta anual es solamente de 80; entonces existe la posibilidad de que el Estado devuelva 20 al contribuyente. En rigor, estos pagos anticipados no se encuentran dentro del concepto legal de tributo.

En cambio un concepto constitucional del tributo bien puede incluir a los referidos pagos definitivos y pagos provisionales, de tal modo que estos últimos también van a recibir cobertura constitucional; es decir, que queda habilitada la posibilidad para que se les apliquen los principios constitucionales tributarios, con la finalidad de que estas cargas económicas sui géneris sean lo más razonable posible.

El segundo párrafo del artículo 74 de la Constitución del Perú de 1993 dice: «El Estado, al ejercer la potestad tributaria, debe respetar los principios de reserva de la ley, y los de igualdad y respeto de los derechos fundamentales de la persona. Ningún tributo puede tener carácter confiscatorio».

Sobre el particular, más allá de la interpretación literal del término «tributo», el Tribunal Constitucional del Perú ha establecido que este vocablo es aplicable a los pagos definitivos y también provisionales que realizan los contribuyentes en favor de Estado, según se aprecia en la sentencia recaída en el expediente 033-2004-AI/TC FJ 8 del 28 de setiembre de 2004, cuando establece lo siguiente:

Este Tribunal considera necesario precisar, más allá de que el tema abordado en esta sección constituye cuestión pacífica, que no existe argumentación valedera que permita sostener que los límites de la potestad tributaria previstos constitucionalmente no son de aplicación a las obligaciones legales derivadas de una obligación tributaria en tanto ellas se estructuran y aplican teniendo en cuenta el tributo al cual se encuentran ligadas; en este caso, al tratarse de un sistema de pagos anticipados que se ejecuta dentro de la estructura del impuesto a la renta - como coinciden demandantes y demandados-, es pertinente que el análisis de la norma cuestionada se someta a los límites a la potestad 
tributaria previstos en la Carta Magna, y sólo desde allí, verificar si nos encontramos ante una norma inconstitucional.

Aquí también es pertinente destacar el importante rol del Tribunal Constitucional en el desarrollo de la definición constitucional del tributo.

\section{IX.6. Control constitucional}

Como sabemos, tradicionalmente el control de la primacía de la Constitución respecto de las normas infraconstitucionales estaba reservado al Poder Judicial. En virtud del proceso de constitucionalización del derecho esta clase de control ahora también está a cargo de órganos especializados como el Tribunal Constitucional, quien asume el denominado control concentrado, de tal modo que el Poder Judicial más bien cumple con la tarea del control difuso.

En el Perú se ha dado un paso más en esta dirección, pues incluso los tribunales administrativos pueden realizar control difuso, según se desprende de la sentencia del Tribunal Constitucional del Perú recaída en el expediente 3741-2004-AA/TC FJ 7 del 14 de noviembre de 2005, que constituye precedente vinculante cuando dispone lo siguiente:

El Tribunal Constitucional estima que la administración pública, a través de sus tribunales administrativos o de sus órganos colegiados, no solo tiene la facultad de hacer cumplir la Constitución — dada su fuerza normativa - sino también el deber constitucional de realizar el control difuso de las normas que sustentan los actos administrativos y que son contrarias a la Constitución o a la interpretación que de ella haya realizado el Tribunal Constitucional (artículo VI del Título Preliminar del Código Procesal Constitucional). Ello se sustenta, en primer lugar, en que si bien la Constitución, de conformidad con el párrafo segundo del artículo 138, reconoce a los jueces la potestad para realizar el control difuso, de ahí no se deriva que dicha potestad les corresponda únicamente a los jueces, ni tampoco que el control difuso se realice únicamente dentro del marco de un proceso judicial.

Ahora bien, retomando la materia tributaria, es conocido que la presencia de los principios constitucionales tributarios es fundamental en la definición del tributo, al punto que una carga económica a favor del Estado, que se encuentra desprovista de estos principios, carece de la identidad ontológica que es propia del «tributo».

Sobre el particular, un caso interesante se presentó con el llamado Impuesto Mínimo a la Renta que las empresas tenían que pagar al Estado en función de sus activos y sin importar los resultados (utilidad o pérdida anual). 
Al respecto, el Tribunal Fiscal del Perú, que es un órgano administrativo, ha ejercido la facultad del control difuso, negando la calidad de tributo a cierta carga económica que la ley denominaba «impuesto» por contravenir el principio constitucional de no confiscatoriedad, criterio que ya había sido adelantado por el Tribunal Constitucional en anteriores casos similares.

En efecto, en la resolución del Tribunal Fiscal 05132-3-2009 del 29 de mayo de 2009, p. 7, se sostiene lo siguiente:

Que el Tribunal Constitucional, en reiterada jurisprudencia tal como las sentencias recaídas en los expedientes 0646-1996-AA/TC, 09172003-AA/TC, 0676-2005-PA/TC, 3859-2004-AA/TCy 4623-2005-PA/ TC, entre otras, ha dejado establecido que el Impuesto Mínimo a la Renta es inconstitucional por violar el principio de no confiscatoriedad de los tributos y que tal inconstitucionalidad radica en que en el seno de una ley, como el decreto legislativo 774, destinada a gravar la renta se termine gravando la fuente productora de la misma, en ese sentido la incongruencia de un medio (el Impuesto Mínimo a la Renta) con los fines que perseguía el legislador tributario (gravar la renta) es lo que en dicho caso se consideró inconstitucional, por lo tanto no importa si la empresa ha generado beneficios ganancias o rentas o si ha incurrido en pérdidas, la aplicación de los artículos del decreto legislativo 774 referidos al Impuesto Mínimo a la Renta no pueden surtir efectos.

Como se aprecia, este Tribunal administrativo también ha participado en el desarrollo del concepto constitucional del tributo. 